\title{
Pien Tze Huang Inhibits Hypoxia-Induced Angiogenesis via HIF-1 $\alpha$ /VEGF-A Pathway in Colorectal Cancer
}

\author{
Hongwei Chen, ${ }^{1,2}$ Jianyu Feng, ${ }^{1}$ Yuchen Zhang, ${ }^{1}$ Aling Shen, ${ }^{1,2}$ Youqin Chen, ${ }^{3}$ \\ Jiumao Lin, ${ }^{1,2}$ Wei Lin, ${ }^{1}$ Thomas J. Sferra, ${ }^{3}$ and Jun Peng ${ }^{1,2,4}$ \\ ${ }^{1}$ Academy of Integrative Medicine, Fujian University of Traditional Chinese Medicine, 1 Huatuo Road, Minhou Shangjie, \\ Fuzhou, Fujian 350122, China \\ ${ }^{2}$ Fujian Key Laboratory of Integrative Medicine on Geriatric, Fujian University of Traditional Chinese Medicine, 1 Huatuo Road, \\ Minhou Shangjie, Fuzhou, Fujian 350122, China \\ ${ }^{3}$ Rainbow Babies \& Children's Hospital, Case Western Reserve University School of Medicine, 11100 Euclid Avenue, \\ Cleveland, OH 44106, USA \\ ${ }^{4}$ Postdoctoral Workstation, Zhangzhou Pien Tze Huang Pharmaceutical Co., Ltd., Shangjie, Zhangzhou, Fujian 363000, China
}

Correspondence should be addressed to Jun Peng; pjunlab@hotmail.com

Received 29 June 2014; Revised 30 October 2014; Accepted 3 November 2014

Academic Editor: Tzeng-Ji Chen

Copyright (C) 2015 Hongwei Chen et al. This is an open access article distributed under the Creative Commons Attribution License, which permits unrestricted use, distribution, and reproduction in any medium, provided the original work is properly cited.

\begin{abstract}
Hypoxia-induced angiogenesis plays an important role in the development and metastasis of solid tumors and is highly regulated by HIF-1 $\alpha$ /VEGF-A pathway. Therefore, inhibiting tumor angiogenesis via suppression of HIF-1 $\alpha$ /VEGF-A signaling represents a promising strategy for anticancer treatment. As a traditional Chinese medicine formula, Pien Tze Huang (PZH) has long been used as a folk remedy for cancer in China and Southeast Asia. Previously, we reported that PZH inhibits colorectal cancer (CRC) growth both in vivo and in vitro. To elucidate the antitumor mechanisms of $\mathrm{PZH}$, in the present study we used human umbilical vein endothelial cells (HUVEC) and colorectal carcinoma HCT-8 cells to evaluate the effects of PZH on hypoxia-induced angiogenesis and investigated the underlying molecular mechanisms. We found that $\mathrm{PZH}$ could inhibit hypoxia-induced migration and tube formation of HUVEC cells in a dose-dependent manner, although the low concentrations of PZH had no effect on HUVEC viability. Moreover, PZH inhibited hypoxia-induced activation of HIF-1 $\alpha$ signaling and the expression of VEGF-A and/or VEGFR2 in both HCT-8 and HUVEC cells. Collectively, our findings suggest that PZH can inhibit hypoxia-induced tumor angiogenesis via suppression of HIF-1 $\alpha$ /VEGF-A pathway.
\end{abstract}

\section{Introduction}

Colorectal cancer (CRC) is the third most commonly diagnosed cancer worldwide and the second leading cause of cancer mortality in the developed world [1]. Approximately one million new cases are diagnosed every year and 500,000 deaths are reported annually [2]. CRC is a disease characterized by activation of multiple signaling pathways which stimulate proliferation and angiogenesis as well as invasion and metastasis [3-5]. During the last decade, the survival rate of CRC has increased due to early diagnosis, staging, and improvements in surgery and therapy $[6,7]$. However, tumor progression to a highly metastatic stage (mCRC) decreases the overall 5 -year survival rate to below $10 \%$, in part due to tumor angiogenesis, which is required for tumor growth and metastasis [8-11].

Angiogenesis is a central mechanism in human colorectal cancer development and growth $[12,13]$. In particular, vascular endothelial growth factor (VEGF) is closely associated with the induction of neovascularization in human colon cancer [14]. VEGF is one of the most important endogenous ligands of receptors present on the endothelial cell plasma membrane and its binding leads to intracellular signaling and, ultimately, gene transcription that promotes endothelial proliferation, migration, and tube formation of endothelial cells resulting in tumor growth and metastasis $[15,16]$. In addition, VEGF is mediated by multiple interacting genetic and environmental signals [17]. A hypoxic microenvironment 
that is critical for the tumorigenic process in general can stimulate VEGF via hypoxia inducible factor-1 (HIF-1), its primary regulator during hypoxic conditions.

HIF-1 is a dimeric transcription factor composed of the HIF- $1 \alpha$ and HIF- $1 \beta$ subunits. HIF- $1 \alpha$ is a key player in the maintenance of cellular homeostasis under hypoxic conditions through its regulation of the expression of many genes involved in a crucial aspect of cancer biology [18-20]. Under normoxic conditions, prolyl hydroxylase mediates the hydroxylation of the proline residues of HIF- $1 \alpha$ at two positions that allow HIF-1 $\alpha$ to interact with the von Hippel-Lindau E3 ligase complex which allows for degradation by the ubiquitinproteasome system [21]. Under hypoxic conditions, HIF-1 $\alpha$ is not degraded and is able to bind with the ubiquitously expressed HIF-1 $\beta$ subunit. The heterodimeric HIF-1 rapidly translocates to the nucleus, where it binds to the hypoxiaresponsive element and regulates several genes responsible for cellular development under hypoxic conditions. One of the HIF-1-responsive genes is VEGF, which promotes angiogenesis in tumors [22].

Owing to the important role of angiogenesis in the progression and metastasis of solid tumors, inhibition of tumor angiogenesis has become a promising strategy for anticancer chemotherapy. Various kinds of antiangiogenic agents are currently being developed. However, redundancy and cross talk in these pathways form a complicated and robust network which is difficult to target. Currently, angiogenesis inhibitors that target only a single pathway may be insufficient and may contribute to drug resistance [23]. These problems highlight the need for the development of novel anticancer agents. Natural products, such as TCM, have been used as therapies for a very long time [24-26]. Pien Tze Huang $(\mathrm{PZH})$ is a well-known TCM formula that was first prescribed 450 years ago in the Ming Dynasty. $\mathrm{PZH}$ has been used in China and Southeast Asia for centuries as a folk remedy for various types of cancer $[27,28]$. We have previously reported that $\mathrm{PZH}$ can inhibit colon cancer cell growth in vitro via promotion of apoptosis [29]. In addition, using a CRC mouse xenograft model, we found that $\mathrm{PZH}$ can suppress tumor growth in vivo without apparent adverse effects and was shown to inhibit vascular endothelial cell proliferation in vitro and capillary tube formation in vivo $[30,31]$. The effects of $\mathrm{PZH}$ on hypoxia-induced angiogenesis during hypoxic conditions have not been explored.

Here, we show that PZH strongly downregulates the expression of HIF- $1 \alpha$ and inhibits angiogenesis in hypoxic conditions. $\mathrm{PZH}$ potently inhibited hypoxia-induced transcription and translation of VEGF-A. Moreover, we found that PZH ameliorates hypoxia-stimulated angiogenesis. These results demonstrate that PZH may inhibit hypoxia-induced angiogenesis by downregulating HIF-1 $\alpha$ /VEGF-A activity.

\section{Materials and Methods}

2.1. Materials and Reagents. All chemicals, unless otherwise stated, were obtained from Sigma-Aldrich (St. Louis, MO, USA). Roswell Park Memorial Institute Medium 1640 (RPMI RPMI-1640), penicillin-streptomycin, fetal bovine serum
(FBS), Trypsin-EDTA, and TRIzol reagent were purchased from Invitrogen (Carlsbad, CA, USA). SuperScript II reverse transcriptase was obtained from Promega (Madison, WI, USA). In vitro angiogenesis assay kit was purchased from Millipore (Billerica, MA, USA). Enzyme linked immune sorbent assay (ELISA) kits of human VEGF-A were obtained from Shanghai Xitang Biological Technology Ltd. (Shanghai, China). HIF-1 $\alpha, \beta$-actin antibodies, and horseradish peroxidase- (HRP-) conjugated secondary antibodies were purchased from Cell Signaling (Beverly, MA, USA).

2.2. Preparation of $P Z H$. $\mathrm{PZH}$ was obtained from and authenticated by the sole manufacturer Zhangzhou Pien Tze Huang Pharmaceutical Co., Ltd., China (Chinese FDA Approval number Z35020242). Stock solution of PZH was prepared immediately prior to use by dissolving the $\mathrm{PZH}$ powder in phosphate buffered saline (PBS) to a concentration of $20 \mathrm{mg} / \mathrm{mL}$.

2.3. Cell Culture. Human umbilical vein endothelial cells (HUVECs) and human colon carcinoma cells (HCT-8) were obtained from Nanjing KeyGen Biotech. Co. Ltd. (Nanjing, Jiangsu, China). HCT-8 and HUVECs cells were grown in RPMI-1640 containing 10\% (v/v) FBS and $100 \mathrm{U} / \mathrm{mL}$ penicillin and $100 \mu \mathrm{g} / \mathrm{mL}$ streptomycin. Cells were cultured at $37^{\circ} \mathrm{C}$, in a $5 \% \mathrm{CO}_{2}$ humidified environment (normoxic). To expose the cells to hypoxia, they were placed in a modular incubator chamber (Thermo Forma, Waltham, MA, USA), which was infused with a mixture of $1 \% \mathrm{O}_{2}, 5 \% \mathrm{CO}_{2}$, and $94 \%$ $\mathrm{N}_{2}$.

2.4. Cell Viability Evaluation. Viability of HUVECs was carried out using a 3-(4,5-dimethylthiazol-2-yl)-2, 5-diphenyltetrazolium bromide (MTT) assay. Briefly, HUVECs were harvested from exponential phase cultures growing in RPMI1640 supplemented with $10 \%$ FBS, counted, plated in 96-well flat-bottomed microtiter plates $(100 \mu \mathrm{L}$ cell suspensions, $6 \times$ $10^{4}$ cells/mL for HUVECs cells), and treated with medium containing various concentrations of $\mathrm{PZH}$. After 24 hours, the media were removed and $100 \mu \mathrm{L}$ of MTT solution $(0.5 \mathrm{mg} / \mathrm{mL}$ in PBS) was added, and the reaction mixture was incubated at $37^{\circ} \mathrm{C}$ in a $5 \% \mathrm{CO}_{2}$ atmosphere for 4 hours. The MTT solution was removed and $100 \mu \mathrm{L}$ of DMSO added. Optical density was measured using a spectrophotometer (Bio-Tek Model ELX800, USA) at $570 \mathrm{~nm}$.

2.5. Wound-Healing Cell Migration Assay. HUVECs were plated at $4 \times 10^{5}$ cells/well on a 6 -well plate in normal culture media and allowed to reach $80-90 \%$ confluency. An injury line with a width of $0.6 \sim 1.0 \mathrm{~mm}$ was made with a sterile pipette tip and cells were rinsed with PBS. The cells were incubated with serum-free medium supplemented with $\mathrm{PZH}$ $(0.0625,0.125$, and $0.25 \mathrm{mg} / \mathrm{mL})$ or medium alone as a control in hypoxia $\left(1 \% \mathrm{O}_{2}\right)$ or normoxia $\left(21 \% \mathrm{O}_{2}\right)$. Wound closure was evaluated 12 hours later with a Leica DMIL inverted microscope (Leica, German).

2.6. Migration Assay. Cell migration assays were performed with transwell cell culture chambers with $8 \mu \mathrm{m}$ pore filters 
(Corning, NY, USA). After treatment with various concentrations of PZH under normoxia for 24 hours, HUVECs cells were trypsinized and resuspended in serum-free RPMI 1640. HUVECs $\left(5 \times 10^{4}\right.$ cells in $200 \mu \mathrm{L}$ of serum-free RPMI 1640) were added to the upper compartment in duplicate filters and RPMI-1640 media containing 10\% (v/v) FBS were used as a chemoattractant in the lower chambers. Cells were allowed to migrate for 12 hours in normoxic/hypoxic conditions. The nonmigrating cells were removed from the upper surface of each transwell by cotton swab. Transwell membranes were then stained with crystal violet. For quantification, the average number of migrating cells per field was assessed by counting 5 random fields under a phase-contrast microscope (Leica, German) at 200x magnification.

2.7. Tube Formation Assay. The HUVECs tube formation assay was performed with an ECMatrix assay kit, following the manufacture's protocol. Briefly, HUVECs cells were seeded in 6-well culture plates at a density of $2 \times 10^{5}$ cells $/ \mathrm{mL}$. After overnight incubation, HUVECs were treated with indicated concentrations of PZH for 24 hours in normoxic conditions, harvested and diluted to $2 \times 10^{5}$ cells/well in $200 \mu \mathrm{L}$ media, seeded into 1:1 ECMatix gel (v/v) coated 48-well plates, and incubated at $37^{\circ} \mathrm{C}$ under normoxic/hypoxic conditions for 6 hours. Tubes were imaged using a phase-contrast inverted microscope (Leica, German) at 100x magnification. The degree of tube formation was quantified by counting the number of tube structures in four randomly chosen fields without overlap.

2.8. ELISA. The secretion level of VEGF was detected by ELISA kit according to the manufacturer's protocol. The results were expressed as fold change in the concentration of VEGF relative to that of cells in normoxia.

2.9. RT-PCR Assay. Total RNA was extracted from cultured cells using the Trizol reagent (TakaRa). cDNA was synthesized from $2 \mu \mathrm{g}$ of total RNA with a Reverse Transcription Kit (TakaRa). The primers used in the RT-PCR reactions were the following: (forward primer) $5^{\prime}$-CTC AAA GTC GGA CAG CCT CA- $3^{\prime}$ and (reverse primer) $5^{\prime}$-CCC TGC AGT AGG TTT CTG CT- $3^{\prime}$ for HIF-1 $\alpha$, (forward primer) $5^{\prime}$-TTT CTG CTG TCT TGG GTG CAT TGG-3' and (reverse primer) $5^{\prime}$ TCT GCA TGG TGA TGT TGG ACT CCT- $3^{\prime}$ for VEGF, and $5^{\prime}$-CTC CAG GGC TTC TTG GTT TTC C- $3^{\prime}$ and (reverse primer) $5^{\prime}$-TTT CAC CAT CTG GTT GGC TGG C- $3^{\prime}$ for $\alpha$ tubulin. The PCR products were separated and visualized on an agarose gel stained with ethidium bromide under UV transillumination.

2.10. Western Blot. Cultured cells were lysed in a buffer containing $40 \mathrm{mM}$ Tris-Cl, $10 \mathrm{mM}$ EDTA, $120 \mathrm{mM} \mathrm{NaCl}$, 0.1\% NP40 , and a protease inhibitor cocktail (CALBIOCHEM). Protein concentration was determined using a bicinchoninic acid (BCA) assay. Denatured proteins were separated by SDS/polyacrylamide gel electrophoresis (PAGE) and transferred to a PVDF membrane (MILLIPORE). The membrane was incubated with 5\% skim milk in TBS containing 0.1\% Tween-20

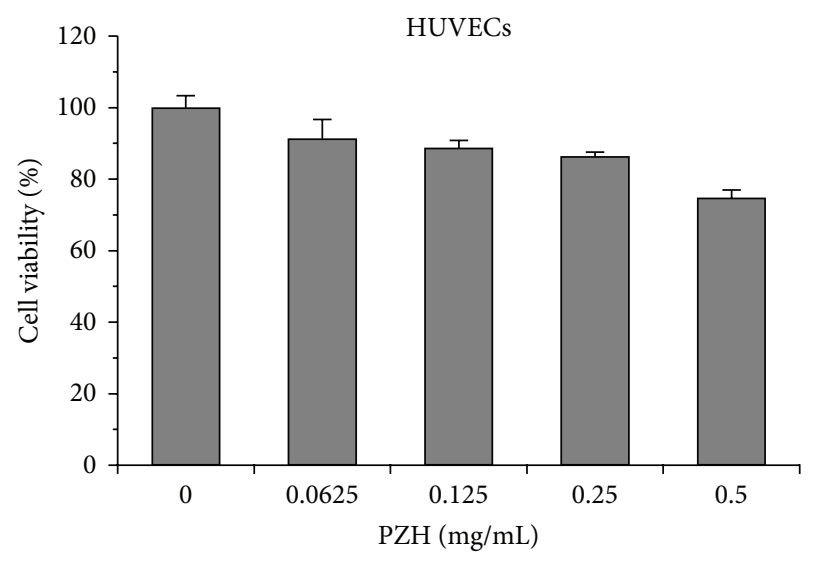

FIgURE 1: The intrinsic cytotoxicity of PZH in HUVECs. HUVECs cells were treated with the indicated concentrations of PZH for $24 \mathrm{~h}$. Cell viability was determined by MTT assay. The data were normalized to the viability of control cells. Data are averages with SD (error bars) from at least three independent experiments.

for 1 hour at room temperature and probed with the appropriate antibodies. The blots were developed using an ECL Prime Detection Kit (Amersham Pharmacia Biotech).

\section{Results}

3.1. Intrinsic Cytotoxicity of $P Z H$ in HUVECs. The lowest dose $(0.0625 \mathrm{mg} / \mathrm{mL})$ of PZH was not cytotoxic to HUVECs, and higher doses $(0.125$ and $0.25 \mathrm{mg} / \mathrm{mL})$ only decreased cell viability by $11.36 \%$ and $13.69 \%$, respectively (Figure 1 ). To investigate angiogenesis inhibition effects of $\mathrm{PZH}$, treatment concentrations of $0.0625-0.25 \mathrm{mg} / \mathrm{mL}$ were chosen.

3.2. PZH Inhibited Hypoxia-Induced Migration of HUVECs. Since endothelial cell migration is a pivotal step for angiogenesis, we performed wound-healing and transwell migration assays in HUVECs pretreated with indicated concentrations of $\mathrm{PZH}$ (Figure 2) [32]. Dose dependent attenuation of hypoxia-induced increase in migration rates into wounds was observed (Figures 2(a) and 2(c)). Hypoxia alone increased transwell migration by 1.64 -fold as compared to normoxia while intervention with $\mathrm{PZH}$ lowered hypoxia-induced increased transwell migration rates to below baseline in a dose dependent manner (Figures 2(b) and 2(d)).

\subsection{PZH Inhibited Hypoxia-Induced Tube Formation of HUV-} ECs. PZH blunted increased capillary-like networks due to hypoxic conditions in a dose dependent manner as well (Figure 3).

3.4. PZH Inhibited Hypoxia-Induced Expression of HIF-1 $\alpha$ and VEGF-A in HCT-8 Cells and HUVECs. HIF- $1 \alpha$ is known to be stabilized during hypoxia driving VEGF expression promoting angiogenesis [33]. We therefore examined whether $\mathrm{PZH}$ could influence the expression patterns of HIF- $1 \alpha$ and VEGFA in HCT-8 and HUVECs cells exposed to hypoxia. PZH suppressed HIF-1 $\alpha$ protein levels as well as mRNA expression in 


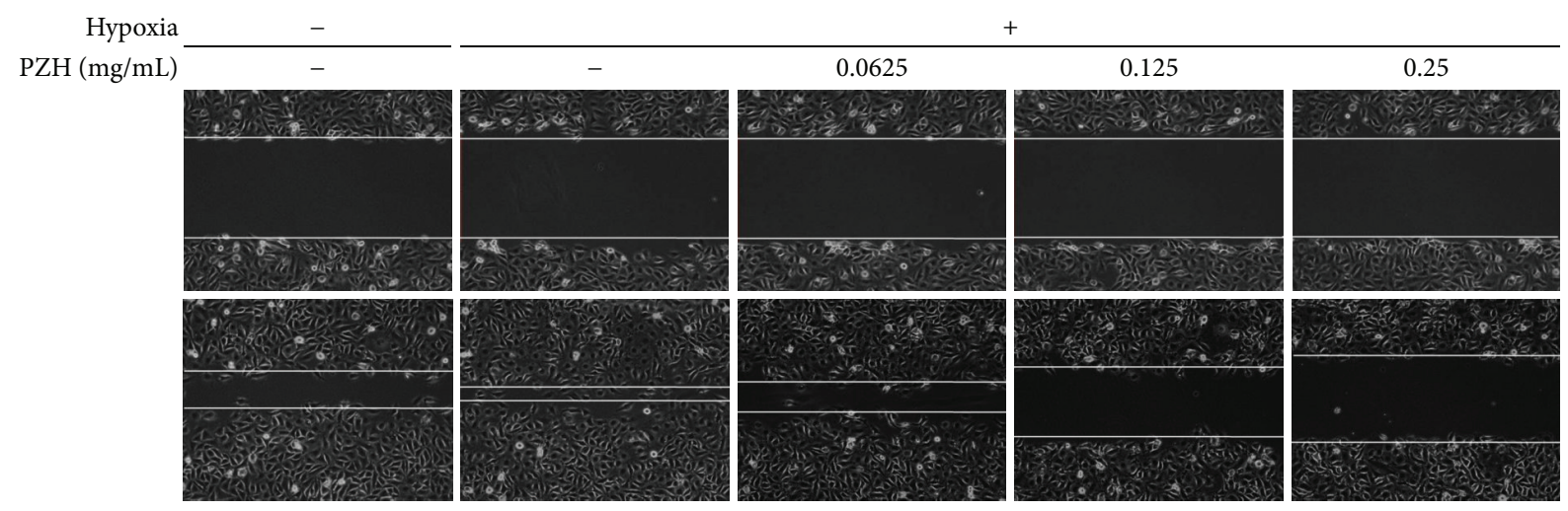

(a)

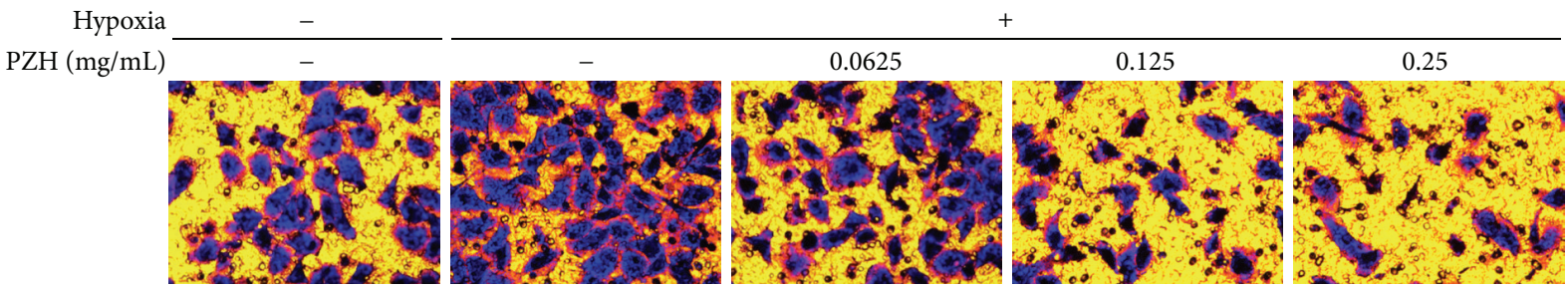

(b)

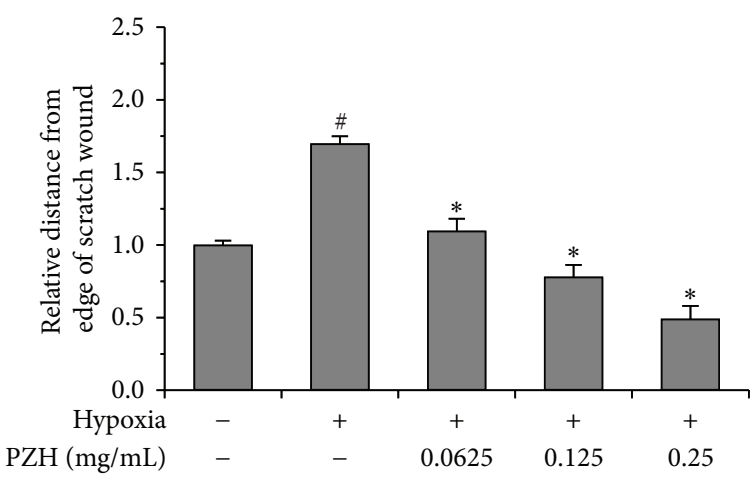

(c)

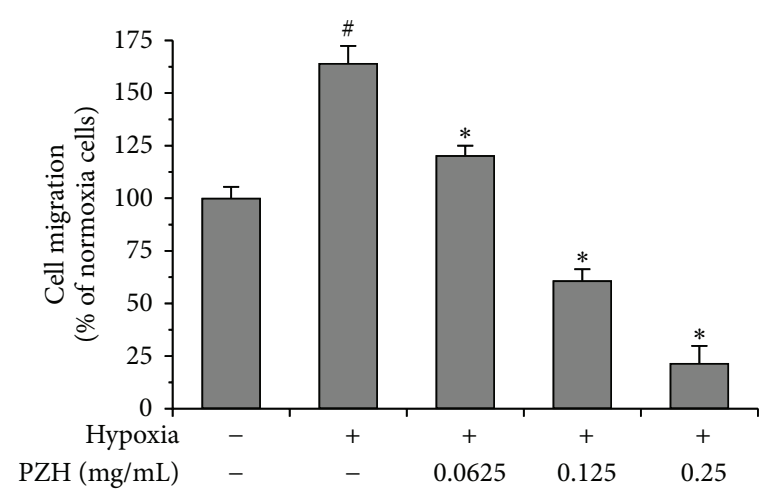

(d)

FIgURE 2: Effect of PZH on the migration in HUVECs. After treatment with the indicated concentrations of PZH for $24 \mathrm{~h}$, the migration pattern of HUVECs was observed using phase contrast microscopy, wound-healing, and transwell migration assays. (a) A confluent monolayer of HUVEC was scratched in the middle with a pipette tip and the scratched area covered by cells that had migrated after exposure to normoxia or hypoxia for $12 \mathrm{~h}$ was measured; photographs are at 100x magnification. Images are representative of three independent experiments. (b) Transwell migration assays of HUVECs. Images are at 100x magnification. Images are representative of three independent experiments. (c) Migration distance after wound relative to the control at time of wound. ${ }^{*}$ A significant difference compared with the normoxic control $(P<0.05)$. *A significant difference compared with the hypoxic control $(P<0.05)$. (d) Measurement of the average number of migrating cells per field was assessed by counting 5 random fields. Statistics are expressed as the mean \pm SD. ${ }^{*}$ A significant difference compared with the normoxic control $(P<0.05) .{ }^{*}$ A significant difference compared with the hypoxic control $(P<0.05)$.

both HUVECs and HCT-8 cells (Figures 4(a), 4(b), 5(a), and 5(b)). Moreover, $\mathrm{PZH}$ inhibited hypoxia-induced secretion of VEGF-A in both cell lines as well (Figures 4(c) and 5(c)). These results suggest that downregulated HIF-1 $\alpha /$ VEGFA pathway by $\mathrm{PZH}$ could inhibit the hypoxia-stimulated migration and tubular formation of endothelial cells.

\section{Discussion}

HIF- $1 \alpha$, a transcription factor, has been demonstrated to be associated with most hypoxic solid tumors and is consistently upregulated in various types of cancers and promotes tumorigenesis through angiogenesis $[19,34,35]$. Aberrant expression of VEGF is known to be a key regulator in hypoxia-induced angiogenesis. Although PZH is known to inhibit VEGF expression via multiple cellular pathways, the effect of $\mathrm{PZH}$ on hypoxia-induced VEGF expression and the regulation of HIF- $1 \alpha$ activity by PZH are not well understood [31]. In this study, we have demonstrated that VEGF, a potent hypoxiainduced angiogenic factor, and HIF- $1 \alpha$, the transcription factor essential for VEGF transcriptional activation, can be regulated by $\mathrm{PZH}$ in HCT-8 and HUVECs. We further 


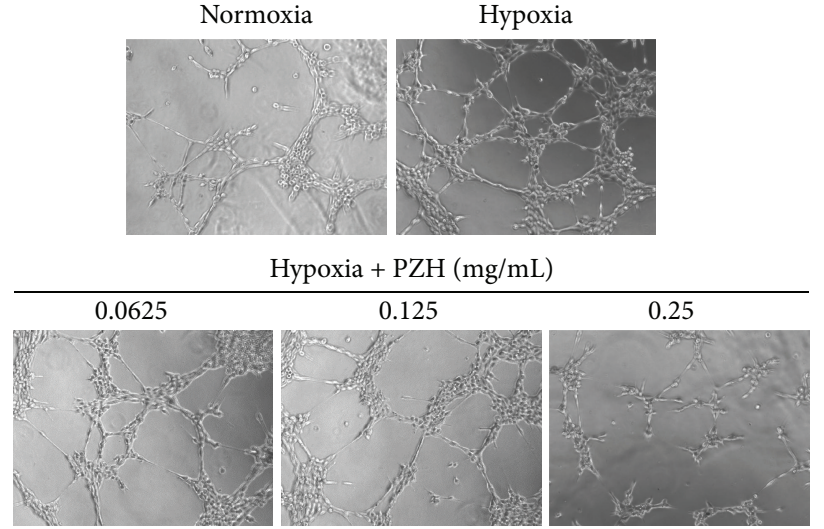

(a)

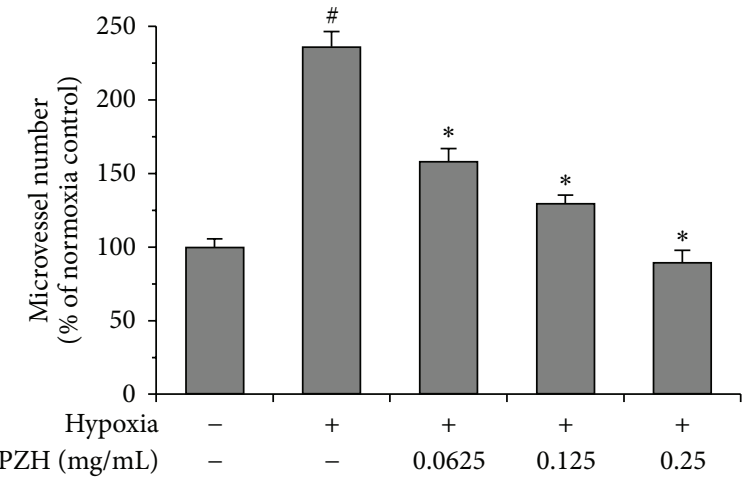

(b)

FIGURE 3: Effects of PZH on tube formation in HUVECs. (a) HUVECs cells were treated with PZH for $24 \mathrm{~h}$, and then collected HUVECs were cultured in Matrigel coated plates. Representative photomicrographs, at 200x magnification, of three independent experiments are shown. (b) The representative histogram of the area covered by the tube network was quantitated using Image-Pro Plus software. ${ }^{\#} \mathrm{~A}$ significant difference compared with normoxic control $(P<0.05) .{ }^{*}$ A significant difference compared with hypoxic control $(P<0.05)$.

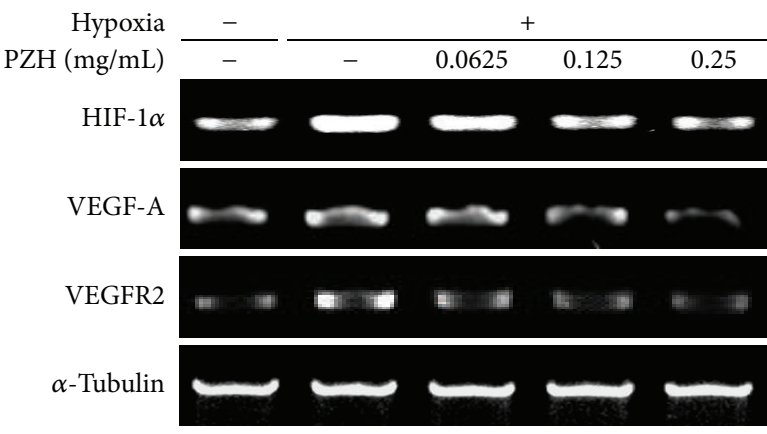

(a)

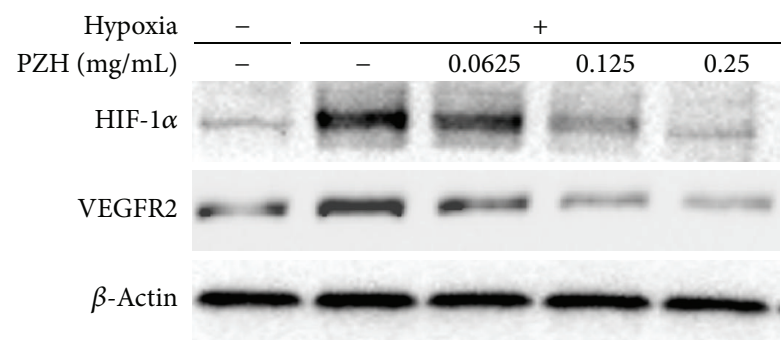

(b)

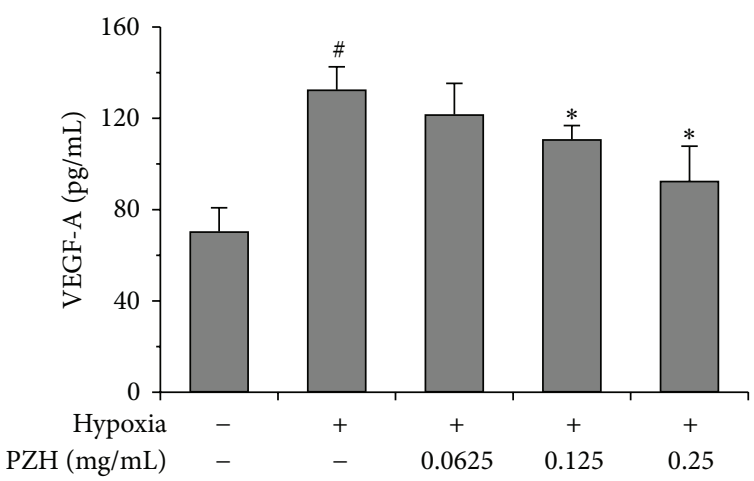

(c)

FIGURE 4: Effects of PZH on HIF-1 $\alpha$, VEGF-A, and VEGFR2 expression in HUVECs. (a) mRNA expression levels of HIF-1 $\alpha$ were analyzed by RT-PCR, and $\alpha$-tubulin was used as a loading control. (b) Protein expression levels of HIF-1 $\alpha$ and VEGFR2 were analyzed by Western blot, and $\beta$-actin was used as a loading control. (c) Secreted VEGF protein was analyzed by ELISA. \#A significant difference compared with normoxic control $(P<0.05) .{ }^{*}$ A significant difference compared with hypoxic control $(P<0.05)$.

revealed that PZH directly suppresses hypoxia-stimulated endothelial migration and tube formation, which is also accompanied by decreased HIF-1 $\alpha$ and VEGF expression levels in PZH-treated HUVECs. A recent report showed that loss of hypoxia-responsive HIF-1 $\alpha$ in endothelial cells results in impaired angiogenesis. This impairment is a direct result of the disruption of an autocrine loop acting through HIF-1 $\alpha$ regulation of hypoxia-induced VEGF expression [33]. Based on our observations in this project, $\mathrm{PZH}$ may play a direct role in the degradation of HIF- $1 \alpha$ and subsequent VEGF signaling 


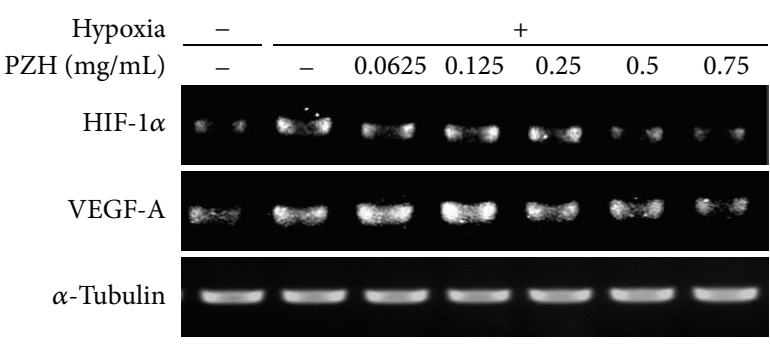

(a)

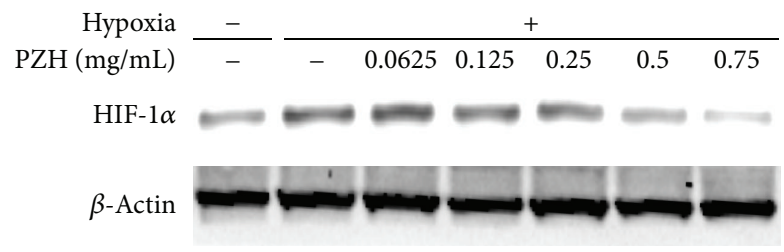

(b)

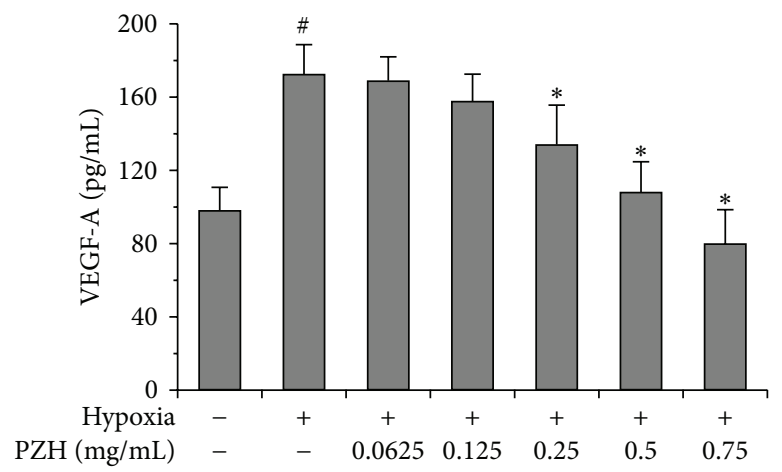

(c)

FIGURE 5: Effects of PZH on the expression of HIF-1 $\alpha$ and VEGF-A in HCT-8. (a) mRNA expression levels of HIF-1 $\alpha$ were analyzed by RT-PCR, and $\alpha$-tubulin was used as a loading control. (b) Protein expression levels of HIF- $1 \alpha$ were analyzed by Western blot, and $\beta$-actin

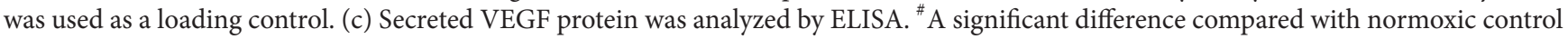
$(P<0.05) .{ }^{*}$ A significant difference compared with hypoxic control $(P<0.05)$.

in colorectal cancer cells and endothelial cells, leading to the inhibition of hypoxia-induced tumor angiogenesis, which is consistent with our previous study that PZH displays antitumor angiogenesis activity in the colorectal cancer mouse xenograft model [31].

Interestingly, many drugs may enhance vascularization in normal injured tissue while doing the reverse in tumor. Although there are no reports directly demonstrating the effects of Pien Tze Huang (PZH) on vascularization in the normal injured tissue, $\mathrm{PZH}$ is believed to be able to promote would healing because it has long been used to clinically treat traumatic injuries. In addition, recently it has been reported that Panax notoginseng saponins (PNS), one of major components in $\mathrm{PZH}$, exerted a significant therapeutic effect on a complex disease condition featuring concomitant presentation of lung carcinoma and myocardial ischemia in mouse, where PNS was able to inhibit tumor angiogenesis and meanwhile promote myocardial ischemia-induced angiogenesis [36]. Therefore, we speculate that $\mathrm{PZH}$ probably also bidirectionally regulates angiogenesis: enhancing vascularization in normal injured tissue while doing the reverse in tumors. Although Yang et al. further proposed that PNS bidirectionally regulated angiogenesis through modulating the expression of proangiogenic miR-18a in a tissue specific manner, they did not address why PNS displayed opposite regulation of angiogenesis and related miRNA expression in different tissues. The possible mechanisms mediating the bidirectional angiogenic activity of PNS and other drugs could be the difference of microenvironment between tumors and normal tissues.
It has been shown that tumor blood vessels differ morphologically from their normal counterparts, which display inappropriate growth and abnormal proliferation and apoptosis in endothelial cells, insufficient pericyte recruitment, enhanced leakiness, decreased blood flow, and abnormalities in the basement membrane [37, 38]. Moreover, gene expression analysis of endothelial cells derived from human blood vessels of normal and malignant colorectal tissues revealed that forty-six genes were specifically elevated in tumorassociated endothelium [39]. Furthermore, hypoxia is a common characteristic feature of all rapidly growing solid tumors, which may trigger the activation of tumor-related signaling pathways such as HIF-1 $\alpha$ [40]. However, the precise mechanisms of how drugs exert bidirectional angiogenic function remain largely unclear. These intriguing and challenging questions must be addressed by future studies before these drugs can be developed as better antiangiogenic agents for cancer treatment.

\section{Conflict of Interests}

The authors declare no financial or commercial conflict of interests.

\section{Acknowledgments}

This work was sponsored by the National Natural Science Foundation of China (81373819 and 81202790) and the China Postdoctoral Science Foundation (2013T60636). 


\section{References}

[1] A. Jemal, R. Siegel, J. Xu, and E. Ward, "Cancer statistics, 2010," A Cancer Journal for Clinicians, vol. 60, no. 5, pp. 277-300, 2010.

[2] E. Chu, "An update on the current and emerging targeted agents in metastatic colorectal cancer," Clinical Colorectal Cancer, vol. 11, no. 1, pp. 1-13, 2012.

[3] Y. Y. Zaytseva, V. A. Elliott, P. Rychahou et al., "Cancer cellassociated fatty acid synthase activates endothelial cells and promotes angiogenesis in colorectal cancer," Carcinogenesis, vol. 35, no. 6, pp. 1341-1351, 2014.

[4] S. Gaur, L. Chen, V. Ann et al., "Dovitinib synergizes with oxaliplatin in suppressing cell proliferation and inducing apoptosis in colorectal cancer cells regardless of RAS-RAF mutation status," Molecular Cancer, vol. 13, no. 1, article 21, 2014.

[5] K. Rupertus, J. Sinistra, C. Scheuer et al., "Interaction of the chemokines I-TAC (CXCL11) and SDF-1 (CXCL12) in the regulation of tumor angiogenesis of colorectal cancer," Clinical \& Experimental Metastasis, vol. 31, no. 4, pp. 447-459, 2014.

[6] N. Kapse and V. Goh, "Functional imaging of colorectal cancer: positron emission tomography, magnetic resonance imaging, and computed tomography," Clinical Colorectal Cancer, vol. 8, no. 2, pp. 77-87, 2009.

[7] O. Majek, A. Gondos, L. Jansen et al., "Survival from colorectal cancer in Germany in the early 21st century," British Journal of Cancer, vol. 106, no. 11, pp. 1875-1880, 2012.

[8] J. M. Davies and R. M. Goldberg, "Treatment of metastatic colorectal cancer," Seminars in Oncology, vol. 38, no. 4, pp. 552560, 2011.

[9] T. Winder and H.-J. Lenz, "Vascular endothelial growth factor and epidermal growth factor signaling pathways as therapeutic targets for colorectal cancer," Gastroenterology, vol. 138, no. 6, pp. 2163-2176, 2010.

[10] J. Folkman, "Tumor angiogenesis: therapeutic implications," The New England Journal of Medicine, vol. 285, no. 21, pp. 11821186, 1971.

[11] J. Folkman and M. Klagsbrun, "Angiogenic factors," Science, vol. 235, no. 4787, pp. 442-447, 1987.

[12] C. A. Staton, A. S. A. Chetwood, L. C. Cameron, S. S. Cross, N. J. Brown, and M. W. R. Reed, "The angiogenic switch occurs at the adenoma stage of the adenoma-carcinoma sequence in colorectal cancer," Gut, vol. 56, no. 10, pp. 1426-1432, 2007.

[13] R. S. Warren, H. Yuan, M. R. Matli, N. A. Gillett, and N. Ferrara, "Regulation by vascular endothelial growth factor of human colon cancer tumorigenesis in a mouse model of experimental liver metastasis," The Journal of Clinical Investigation, vol. 95, no. 4, pp. 1789-1797, 1995.

[14] Y. Takahashi, Y. Kitadai, C. D. Bucana, K. R. Cleary, and L. M. Ellis, "Expression of vascular endothelial growth factor and its receptor, KDR, correlates with vascularity, metastasis, and proliferation of human colon cancer," Cancer Research, vol. 55, no. 18, pp. 3964-3968, 1995.

[15] A. M. Byrne, D. J. Bouchier-Hayes, and J. H. Harmey, "Angiogenic and cell survival functions of vascular endothelial growth factor (VEGF)," Journal of Cellular and Molecular Medicine, vol. 9, no. 4, pp. 777-794, 2005.

[16] X.-Y. Wang, S. Ju, C. Li et al., "Non-invasive imaging of endothelial progenitor cells in tumor neovascularization using a novel dual-modality paramagnetic/near-infrared fluorescence probe," PLoS ONE, vol. 7, no. 11, Article ID e50575, 2012.

[17] H. Hurwitz, L. Fehrenbacher, W. Novotny et al., "Bevacizumab plus irinotecan, fluorouracil, and leucovorin for metastatic colorectal cancer," The New England Journal of Medicine, vol. 350, no. 23, pp. 2335-2342, 2004.

[18] M. C. Brahimi-Horn, J. Chiche, and J. Pouysségur, "Hypoxia and cancer," Journal of Molecular Medicine, vol. 85, no. 12, pp. 13011307, 2007.

[19] G. L. Semenza, "HIF-1 and tumor progression: pathophysiology and therapeutics," Trends in Molecular Medicine, vol. 8, no. 4, pp. S62-S67, 2002.

[20] G. L. Semenza, "Hypoxia-inducible factors: mediators of cancer progression and targets for cancer therapy," Trends in Pharmacological Sciences, vol. 33, no. 4, pp. 207-214, 2012.

[21] S. Kizaka-Kondoh, S. Tanaka, H. Harada, and M. Hiraoka, “The HIF-1-active microenvironment: an environmental target for cancer therapy," Advanced Drug Delivery Reviews, vol. 61, no. 7-8, pp. 623-632, 2009.

[22] S. A. Stacker, C. Caesar, M. E. Baldwin et al., "VEGF-D promotes the metastatic spread of tumor cells via the lymphatics," Nature Medicine, vol. 7, no. 2, pp. 186-191, 2001.

[23] H. P. Eikesdal and R. Kalluri, "Drug resistance associated with antiangiogenesis therapy," Seminars in Cancer Biology, vol. 19, no. 5, pp. 310-317, 2009.

[24] D. J. Newman, G. M. Cragg, and K. M. Snader, "The influence of natural products upon drug discovery," Natural Product Reports, vol. 17, no. 3, pp. 215-234, 2000.

[25] C. Spatafora and C. Tringali, "Natural-derived polyphenols as potential anticancer agents," Anti-Cancer Agents in Medicinal Chemistry, vol. 12, no. 8, pp. 902-918, 2012.

[26] H.-F. Ji, X.-J. Li, and H.-Y. Zhang, "Natural products and drug discovery: can thousands of years of ancient medical knowledge lead us to new and powerful drug combinations in the fight against cancer and dementia?" EMBO Reports, vol. 10, no. 3, pp. 194-200, 2009.

[27] Y. Xu and E. Yu, "Clinical analysis of the effect of Pien Tze Huang in treatment of 42 patients with moderate or advanced liver cancer," Shanghai Journal of Tradition Chinese Medicine, vol. 12, pp. 4-5, 1994.

[28] Z. Gu, "Therapeutical observation of advanced colon cancer," Chinese Traditional Patent Medicine, vol. 15, p. 23, 1993.

[29] J. M. Lin, L. H. Wei, Y. Q. Chen et al., "Pien Tze Huang induced apoptosis in human colon cancer HT-29 cells is associated with regulation of the Bcl-2 family and activation of caspase 3," Chinese Journal of Integrative Medicine, vol. 17, pp. 685-690, 2011.

[30] A.-L. Shen, F. Hong, L.-Y. Liu et al., "Effects of Pien Tze Huang on angiogenesis in vivo and in vitro," Chinese Journal of Integrative Medicine, vol. 18, no. 6, pp. 431-436, 2012.

[31] A. Shen, J. Lin, Y. Chen et al., "Pien Tze Huang inhibits tumor angiogenesis in a mouse model of colorectal cancer via suppression of multiple cellular pathways," Oncology Reports, vol. 30, no. 4, pp. 1701-1706, 2013.

[32] Y. Song, F. Dai, D. Zhai et al., "Usnic acid inhibits breast tumor angiogenesis and growth by suppressing VEGFR2-mediated AKT and ERK1/2 signaling pathways," Angiogenesis, vol. 15, no. 3, pp. 421-432, 2012.

[33] N. Tang, L. Wang, J. Esko et al., "Loss of HIF-1 $\alpha$ in endothelial cells disrupts a hypoxia-driven VEGF autocrine loop necessary for tumorigenesis," Cancer Cell, vol. 6, no. 5, pp. 485-495, 2004.

[34] D. E. Richard, E. Berra, and J. Pouysségur, "Angiogenesis: how a tumor adapts to hypoxia," Biochemical and Biophysical Research Communications, vol. 266, no. 3, pp. 718-722, 1999. 
[35] G. L. Semenza, "HIF-1: using two hands to flip the angiogenic switch," Cancer and Metastasis Reviews, vol. 19, no. 1-2, pp. 5965, 2000.

[36] Q. Yang, X. Wang, J. Cui et al., "Bidirectional regulation of angiogenesis and miR-18a expression by PNS in the mouse model of tumor complicated by myocardial ischemia," $B M C$ Complementary and Alternative Medicine, vol. 14, article 183, 2014.

[37] R. K. Jain, "Molecular regulation of vessel maturation," Nature Medicine, vol. 9, no. 6, pp. 685-693, 2003.

[38] P. Baluk, S. Morikawa, A. Haskell, M. Mancuso, and D. M. McDonald, "Abnormalities of basement membrane on blood vessels and endothelial sprouts in tumors," The American Journal of Pathology, vol. 163, no. 5, pp. 1801-1815, 2003.

[39] B. St. Croix, C. Rago, V. Velculescu et al., "Genes expressed in human tumor endothelium," Science, vol. 289, no. 5482, pp. 1197-1202, 2000.

[40] H. Kimura, A. Weisz, T. Ogura et al., "Identification of hypoxiainducible factor 1 ancillary sequence and its function in vascular endothelial growth factor gene induction by hypoxia and nitric oxide," The Journal of Biological Chemistry, vol. 276, no. 3, pp. 2292-2298, 2001. 


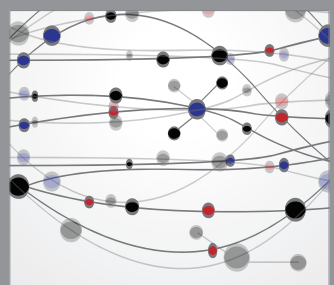

The Scientific World Journal
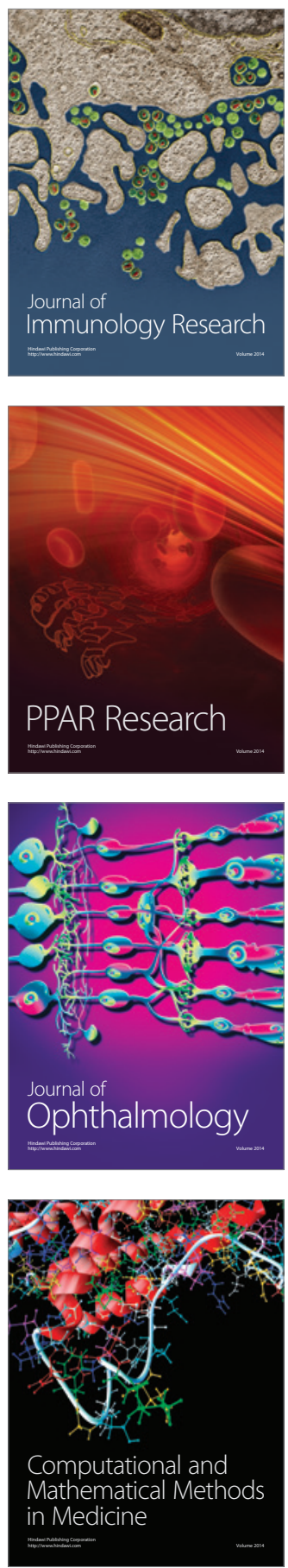

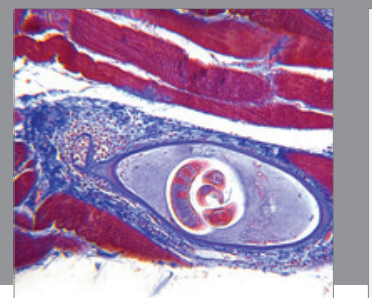

Gastroenterology

Research and Practice
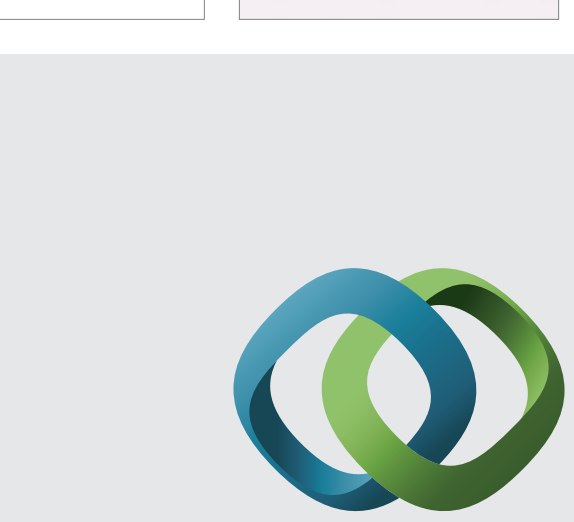

\section{Hindawi}

Submit your manuscripts at

http://www.hindawi.com
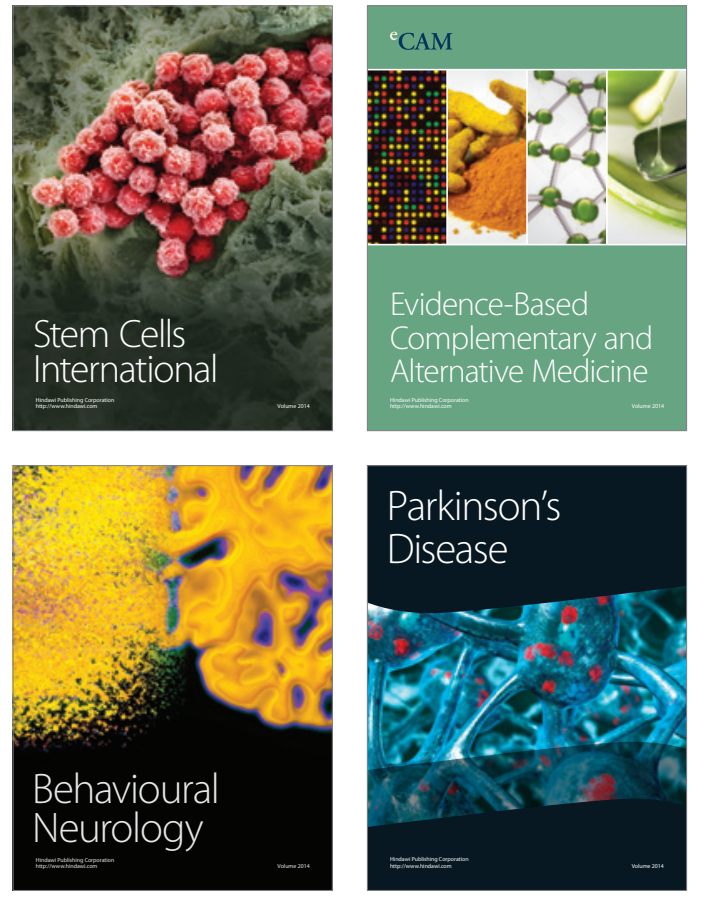
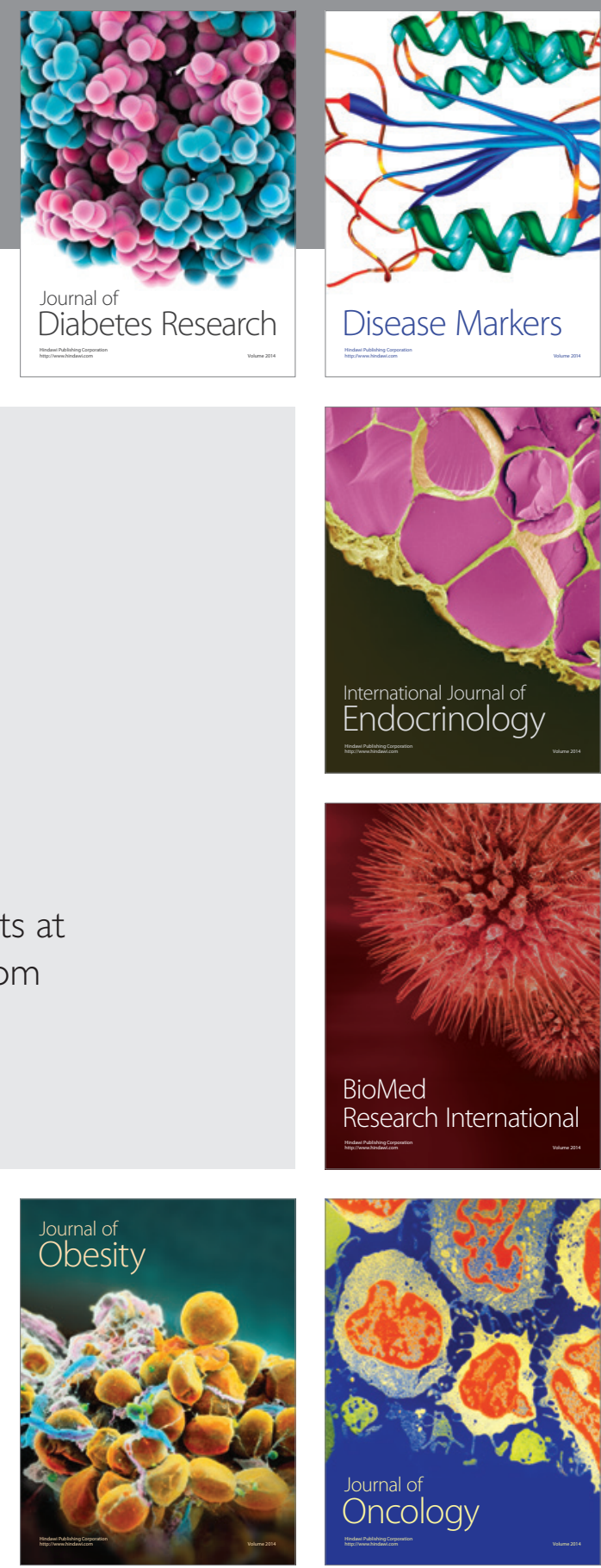

Disease Markers
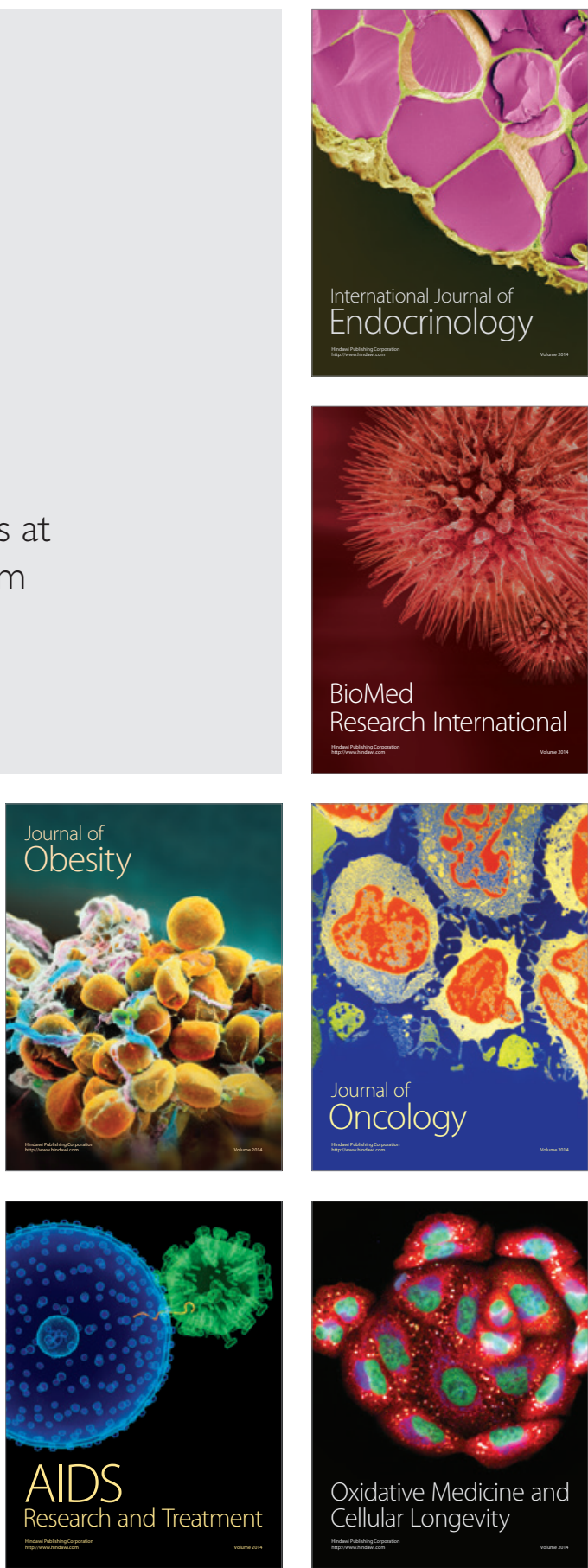